$63^{\text {ème }}$ Congrès de la SFCO, 02002 (2015)

DOI:10.1051/sfco/20156302002

(C) Owned by the authors, published by EDP Sciences, 2015

\title{
COMMUNICATION
}

\section{Apport d'un logiciel de reconstruction 3D dans la prise en charge chirurgico-orthodontique de la dysplasie cléido-crânienne : à propos d'un cas clinique}

\author{
Costa Mendes L, Bodard A-G \\ Service de Consultations et Traitements Dentaires, 69007 Lyon
}

La dysplasie cléido-crânienne est un syndrome décrit pour la première fois par Martin en 1765, caractérisé par une atteinte de l'embryogenèse du tissu osseux et cartilagineux prédominant au niveau du crâne et du thorax (Teyton et al. 1984). Il s'agit d'une maladie héréditaire autosomique dominante, peu fréquente, de l'ordre de 1/1000000, mais présentant une expressibilité phénotypique variable (Cooper et al. 2001). Le diagnostic de la pathologie est confirmé par une analyse génétique recherchant une mutation du gène RUNX2 sur le bras court du chromosome 6 (Mundlos 1997).

Les symptômes les plus fréquemment rencontrés sont une hypoplasie claviculaire, un retard ou absence de soudure des os du crâne et des anomalies au niveau de la cavité orale, en particulier une hypoplasie maxillaire, un retard d'exfoliation des dents temporaires, une absence ou un retard d'éruption des dents permanentes et la présence de multiples dents surnuméraires.

Actuellement, la prise en charge bucco-dentaire de ces patients se fait au sein d'une équipe pluridisciplinaire chirurgico-orthodontique.

Nous rapportons ici un cas de dysplasie cléido-crânienne suivi en chirurgie orale et orthopédie dentofaciale depuis 2012. La difficulté pour cette patiente, âgée de 21 ans, a été la planification du plan de traitement orthodontique, notamment l'établissement de la chronologie des désinclusions. Comme c'est souvent le cas pour ces patients, le challenge réside dans le processus de mise en place des dents permanentes sur l'arcade, compliqué par la présence de dents surnuméraires et par une absence d'ancrage, nécessaire à leur traction. Le scanner $\mathrm{X}$ est l'examen de choix pour la planification des différentes tractions orthodontiques et extractions des dents surnuméraires. Cependant, il n'est pas toujours aisé de se représenter mentalement la position de chaque élément dentaire ainsi que de ses rapports avec les structures environnantes lors de l'analyse des coupes scanner. L'utilisation d'un logiciel de reconstruction tridimensionnelle (3D NeoVision) a permis, dans un premier temps, d'établir un plan de traitement orthodontique en fonction de la position et de l'anatomie des dents permanentes incluses. La prédictibilité du traitement est augmentée puisqu'il est possible de détecter en amont des caractéristiques dentaires défavorables telles une malformation coronaire, une zone d'ankylose, ou encore une proximité importante ou fusion entre une couronne et les racines d'une dent adjacente. Dans un deuxième temps, l'analyse des reconstructions 3D a permis de planifier de façon précise les étapes chirurgicales (extraction de dents surnuméraires, désinclusionset tractions chirurgico-orthodontiques) afin d'être le moins invasif et délabrant possible.

L'utilisation de ce logiciel a également servi d'outil pédagogique, puisqu'il facilite l'exposition du plan de traitement au patient, donc sa compréhension et sa coopération sur le long terme.

This is an Open Access article distributed under the terms of the Creative Commons Attribution License 4.0, which permits unrestricted use, distribution, and reproduction in any medium, provided the original work is properly cited. 
$63^{\text {ème }}$ Congrès de la SFCO

Nom et adresse du conférencier

Leonor COSTA MENDES

Service de Consultations et Traitements Dentaires

6-8 place Depéret

69007 Lyon (France)

leonor.c.mendes@gmail.com 